\title{
Civilisations
}

Revue internationale d'anthropologie et de sciences

humaines

43-2 | 1996

Problèmes africains contemporains

\section{Le forgeron qui devint roi}

Idéologie politique de la chefferie songye des Kalebwe (Zaïre)

\section{Christophe Anthoine}

\section{(2) OpenEdition}

12 Journals

\section{Édition électronique}

URL : http://journals.openedition.org/civilisations/1563

DOI : 10.4000/civilisations. 1563

ISSN : 2032-0442

Éditeur

Institut de sociologie de l'Université Libre de Bruxelles

\section{Édition imprimée}

Date de publication : 1 avril 1996

Pagination : 15-44

ISBN : 2-87263-160-7

ISSN : 0009-8140

\section{Référence électronique}

Christophe Anthoine, «Le forgeron qui devint roi », Civilisations [En ligne], 43-2 | 1996, mis en ligne le 29 juin 2009, consulté le 30 avril 2019. URL : http://journals.openedition.org/civilisations/1563 ; DOI : 10.4000/civilisations. 1563 


\section{Le forgeron qui devint roi \\ Idéologie politique de la chefferie songye des Kalebwe (Zaïre)}

. 
centraux en est un exemple particulièrement célèbre qui a attiré l'attention de nombreux spécialistes de la région. On connaissait moins jusqu'il y a peu la production politico-littéraire de leurs voisins septentrionaux, les Songye. La diffusion récente d'une partie importante de celle-ci permet à présent de s'y intéresser. Mon propos sera de montrer l'unité formelle de cette production dans un cadre régional; cette unité formelle n'implique pas pour autant une unité de discours comme l'illustre notamment le fait que les Songye se servent d'éléments récurrents de l'imaginaire politique de cette région d'Afrique, les figures du chasseur et du forgeron, pour rendre compte de leur conception tout à fait originale du pouvoir.

Célèbre pour ses productions artistiques, et en particulier pour ses fameux masques striés bifwebe, la société songye se distingue par un système politique original. Située dans le sud-est du Zaïre, elle est constituée d'une vingtaine de chefferies indépendantes qui possèdent en commun un principe d'organisation politique fondé sur le partage du pouvoir : après quelques années d'exercice, le titulaire d'une charge politique cède la place à son successeur, issu d'un autre groupe de parenté, de telle manière qu'au fil du temps les différents groupes de parenté constitutifs de la chefferie accèdent chacun à leur tour au pouvoir. Un autre trait distinctif des structures politiques songye est la division de la société en deux catégories; l'une, celle des bamfumu, exerce le pouvoir fondé par un étranger arrivé autrefois dans la chefferie, l'autre, dont la dénomination est variable selon les chefferies mais dont le dignitaire principal est toujours le cite, représente les premiers occupants du sol. Les relations entre ces deux catégories sont complémentaires mais hiérarchisées : en échange de dons qui sont autant de marques de reconnaissance de leurs droits, les premiers occupants du sol intronisent les bamfumu dans un statut plus prestigieux que le leur.

Il convient cependant de noter que, dans la pratique, cette partition de la société est plus idéologique que réelle, le système d'accès à ces catégories faisant que celui-ci relève davantage de la 
stratégie de groupe (par exemple l'aîné accède au bufumu et le cadet à l'autre catégorie) que d'un déterminisme généalogique.

Selon les chefferies, les Songye rendent compte de l'origine de cette structure politique par l'arrivée d'un chasseur, d'un guerrier ou d'un forgeron dans la communauté établie par les premiers occupants du sol. Cet étranger, perçu comme un luba', fonde alors par un exploit guerrier, par la ruse ou en initiant un cycle de dons un ordre politique basé sur la complémentarité entre les représentants des premiers occupants et ceux des immigrants luba.

La plupart des chefferies songye relatent cette origine de façon très sommaire; ce n'est cependant pas le cas des Eki (Fairley 1978, 110-46) et des Kalebwe ${ }^{2}$. chez qui l'on a recueilli des récits sensiblement plus longs et plus étoffés de cette origine ${ }^{3}$.

En 1976, Dunja Hersak a publié le mythe d'origine politique des Kalebwe; il s'agit de la traduction d'un manuscrit songye rédigé par Kitumbika Ngoy qui accéda au titre de chef suprềme des Kalebwe. Je donne ici une traduction française de

${ }^{1}$ Luba est l'ethnonyme dont se réclament plusieurs populations voisines des Songye. On distingue les Luba orientaux (les Luba matrilinéaires de l'est du Shaba, aussi appelés Luha-Hemba), les Luba centraux (les Luba patrilinéaires du Shaba regroupés en un grand royaume, parfois appelés Luba-Shankadi) et les Luba occidentaux (les Luba patrilinéaires situés au Kasaï et qui ne faisaient pas partie du royaume luba, aussi appelés Luba-Kasaï). En parlant des Luba, les Songye font généralement référence aux Luba centraux, réservant l'exoethnonyme de Bambu aux Luba occidentaux.

2 Ces deux chefferies sont aussi les deux chefferies songye les plus importantes par la taille, tant spatiale que démographique (Boone 1961, 37-8, 52$5 \& 215-7)$.

3 Le récit de la chefferie songye des Bena Kayaye publié par Moeller (1936, 143-51) est unc longue généalogie mythique qui rend compte de l'origine des différentes populations de la région, et non pas de l'origine des structures politiques; cette dernière est racontée de façon beaucoup plus brève à l'image de ce que l'on fait dans les chefferies voisines (Mutimiti Mukonkole et al., Kongolo, le 19 septembre 1993). 
l'intégralité de ce texte, d'une part, parce qu'il est devenu difficile d'accès et, d'autre part, pour que le lecteur puisse toujours replacer les éléments que je vais analyser dans le contexte global du mythe car, bien entendu, ma réflexion n'en épuise pas le propos.

L'histoire des chefs kalebwe commence avec l'ancêtre Kiobobo. Celui-ci eut trois fils : Mutombo, Ndala et Kalombo; quand ceux-ci furent grands, Kiobobo qui était chasseur, forgeron et ngang $a^{4}$ leur donna à choisir à chacun une de ces professions. Mutombo choisit le métier de forgeron, Ndala celui de chasseur et Kalombo celui de nganga.

Mutombo Kiobobo, qui eut deux fils (Ilunga et Kimboko), partit à la recherche de nouvelles terres et s'installa sur les rives du lac Moëro. Quand le temps fut venu pour ses fils de choisir à leur tour leur profession, Ilunga décida de collecter les bilase (coquillages blancs en forme d'hélice) et les mambele (cauris) et devint "un chasseur d'eau qui ramasse ce que l'eau vomit" tandis que Kimboko reprit le métier de forgeron de son père. Un jour qu'il se baignait, Kimboko trouva un kilase que son père lui dit de conserver comme une chose propre aux chefs; c'est en vain que son frère le réclama au nom des droits de sa profession. Malheureux et furieux à la fois, ce dernier partit alors, suivant la rivière Lomami jusqu'à Samba.

Avant de mourir, Ilunga, qui n'eut pas d'enfants, chargea Kitumba, un de ceux qui l'accompagnaient, de la garde de ses gens et de ses biens. Il dit aussi à Kitumba que si un jour quelqu'un venait de leur pays d'origine et montrait qu'il était le fils de Kimboko, il devait lui donner tous ses biens. Kimboko, de son côté,

4 Terme qui désigne le magicien ou l'anti-sorcier chez les Bantou. 
eut un fils, Kitenge, qui quitta à son tour le lac Moëro pour s'installer à Kuibwe dia Kitenge. Deux ${ }^{5}$ des Bena Kantu a Muasa y étaient déjà installés : Muilombe Makulo et Kaswa Makulo. Ceux-ci cultivaient déjà certaines plantes mais ils ne possédaient pas d'outils en fer. Alors Kitenge leur forgea des houes, des herminettes, des lances et des flèches et il leur donna aussi de nouvelles plantes à cultiver. Avec leurs nouvelles armes, les Bena Kantu a Muasa allèrent à la chasse et ramenèrent une antilope qu'ils offrirent à Sendwe Kitenge, autrement dit au forgeron Kitenge. N'ayant pas de femme, il en prit une petite partie qu'il donna à Muilombe pour que Kantu a Muasa la lui prépare et il offrit le reste de la viande aux chasseurs. Kitenge partit alors en forêt pour extraire du vin de palme; il le rapporta aux villageois et ceux-ci s'enivrèrent.

Une nuit, Kitenge alla dire à Muilombe qu'il avait faim. Ce dernier demanda à sa femme de préparer quelque chose et quand Kantu a Muasa apporta la nourriture à Kitenge, celui-ci la demanda en mariage. Muilombe alla discuter de l'incident avec Kaswa. Ils se sentaient honteux vis-à-vis de Kitenge qui leur avait apporté des outils et des plantes à cultiver et qui leur avait appris à vivre. Muilombe décida de donner sa première femme, Kantu a Muasa, fille de Muasa a Kamina, à Kitenge. Leurs parents s'étaient opposés à ce mariage parce qu'ils étaient de la même famille et c'est ce qui avait amené Muilombe et Kantu a Muasa à quitter leur patrie.

Kitenge et Kantu a Muasa eurent un fils, Ilunga, et deux filles, Kitoto et Kiseme. 12).

5 Je traduis ainsi l'expression "two people" utilisée par Dunja Hersak (1986, 
Kitenge dit à ses gens qu'il voulait construire une epata ${ }^{6}$ et que ce serait à ebwe (rocher). Sendwe Kitenge devint ainsi Yakitenge Kuibwe ${ }^{7}$. Pour son investiture, il ordonna le sacrifice et la préparation d'un agneau. Mubwangie Mwadi refusa de manger car elle voulait d'abord savoir d'où venait Kitenge. Comme celui-ci avait déjà vécu avec eux depuis un certain temps, Kaswa, qui avait été envoyé pour l'interroger, décida qu'il ne pouvait le lui demander et rapporta que Kitenge était un Luba; comme ces derniers s'étaient dispersés comme des vagabonds, tout le monde fut satisfait. Pour la femme qu'il lui avait offerte, Kitenge donna à Muilombe le nom de Kimungu Sambi (Kimungu: titre du cite). Ainsi les hommes surent que pour devenir babikale ${ }^{8}$. ils devaient suivre l'exemple de Muilombe. Kaswa donna alors un esclave à Kitenge qui changea son nom pour Kiana kia Ngombe (Kiana Ngombe : titre du dipumba, un dignitaire mubikale).

La forge (eyaso) de Kitenge fut construite à l'epata afin qu'il puisse fabriquer outils et armes. La première lance qu'il réalisa fut placée en haut de l'ebwe et la seconde en bas. Kitenge planta alors l'arbre nkabokabo au pied de la forge où il enterra vivante sa fille Kiseme (afin de préserver sa dignité).

Ilunga, en colère à cause du destin de sa soeur, fut banni par son père et se rendit alors à Kilushi où il trouva les gens du chef Luengiela en guerre avec les Buki bua Musongo Ndala. Ilunga les aida en leur montrant comment forger et combattre avec des lances, des arcs et des flèches. Les gens de Luengiela vinrent à bout des Buki et Ilunga prit en charge leur village dont il changea le nom.

6 Ou ehata, résidence du chef suprême et siège du gouvernement central.

7 Titre du chef suprême chez les Kalebwe.

8 Titre générique des dignitaires représentant les premiers occupants du sol. 
Par la suite, Ilunga prépara les villageois à se battre contre les Bahemba. Ils les conquirent et s'installèrent dans leur pays. Là, Ilunga épousa Nabasalemba et en eut deux fils : Ilunga a Ilunga Mbidi Kiluwe et Nkongolo Mwana, et deux filles : Mbu Lemba et Mulo Lemba.

Quand Ilunga mourut, son fils aîné, Ilunga a Ilunga lui succéda comme chef mais les Bahemba lui demandèrent de retourner au pays d'origine de son père, de l'autre côté du fleuve Lualaba. Ilunga a Ilunga laissa à ses frères et soeurs la garde de leurs gens et de leurs biens et partit avec Kinyema, un ancien. Ils suivirent le Lualaba, dépassèrent la route de Kilushi, puis bifurquèrent pour retrouver le fleuve Lomami et arriver à Samba a Kumusienge (Baluba Kasongo Niembo). Ilunga a Ilunga y trouva Kitumba qui, quand il apprit que les ancêtres de cet homme étaient des enfants d'llunga a Kitenge a Kimboko a Mutombo Kiobobo, cria de joie et appela tous les hommes pour qu'ils saluent leur chef. Kitumba dit à Ilunga a Ilunga que tous les gens et le pays de Samba lui appartenaient car ce pays avait été laissé par son grand-père Ilunga a Mutombo Kiobobo.

Un jour, Nkongolo Mwana partit à la recherche de son frère Ilunga a Ilunga. Arrivé dans les environs de Samba, il se cacha près de l'eau mais les femmes de son frère le virent en allant chercher de l'eau et réunirent les deux frères. Ilunga a Ilunga expliqua à son frère comment il en arriva à hériter du pays de Samba et le chargea de continuer à chercher Kuibwe dia Kitenge. Si il y trouvait leur grand-père Kitenge a Kimboko toujours en vie, il devait aller rechercher ses soeurs, Mbu Lemba avec son fils Mpibwe ${ }^{9}$ Kitenge et Mulo Lemba avec son fils Ngoy Mulo, mais s'il le trouvait

${ }^{9}$ Mpibwe signifie chasseur en songye. 
mort, il devait rester là en tant que chef. Arrivé là-bas, Nkongolo Mwana apprit que Yakitenge Kuibwe était mort et que c'était désormais Nsomwe Kiseme, leur neveu, qui régnait, Kaka Kitoto (titre de Kitoto Kitenge, sa grand-mère) l'ayant fait Yakitenge de l'epata avant de partir pour Ekungu où elle devint chef.

Nkongolo Mwana dit à Nsomwe qu'il n'avait pas le droit de régner car il était le fils d'une femme, de sa soeur (classificatoire) Kiseme, et lui ordonna de partir. Nsomwe refusa, rappelant à Nkongolo qu'il était le fils d'Ilunga Kitenge, banni par son père. Nsomwe proposa alors une épreuve où, lançant chacun une lance, celui qui la planterait le plus profondément dans le sol remporterait le pays.

Pendant la nuit, Nsomwe fit creuser un trou à ses hommes et y plaça sa lance. Parachevant la tâche, ils couvrirent le trou d'une natte sur laquelle ils jetèrent de la terre. Par chance, il plut un peu plus tard et toute trace fut effacée.

Le lendemain, Nsomwe apparut avec une calebasse décorée de clous de cuivre attachées à ses parties génitales. Nkongolo Mwana jeta sa lance le premier mais sans succès. Nsomwe prit son élan à son tour, courant et levant haut ses genoux. Quand les gens virent la calebasse attachée entre ses jambes, ils crièrent, trouvant cela extraordinaire. Son chanteur lui chanta «tala potadila, tala potadila (regarde où tu étais en train de regarder), Nsomwe s'approcha du trou et y enfonça entièrement sa lance. Nsomwe Kiseme était victorieux et, comme résultat du miracle, il reçut le nom de Nsomwe Olengiele. Ses gens étaient heureux mais une bataille éclata entre ses hommes et ceux de Nkongolo, au cours de laquelle les villages de Mukombo et Mukuku furent détruits. Défaits, Nkongolo et ses hommes rejoignirent Ilunga à Samba, mais ce dernier 
était furieux et le renvoya chez les Bahemba. Nkongolo mourut en route et fut enterré près du fleuve Lualaba.

Mpibwe Kitenge succéda à Nkongolo et, avec son cousin Ngoy Mulo, il partit à son tour à la recherche du pays de son grand-père Ilunga Kitenge. Ils atteignirent Kilushi et furent bien reçus par les gens de tous les villages songye car les gens disaient "ici est l'enfant de notre chef qui nous a libéré de l'esclavage des Buki". Mpibwe Kitenge remplaça les vieux chefs songye (qui avaient été avec Buki) par des hommes à lui qu'il connaissait mieux. Ensuite il traversa le fleuve Lomami au confluent de celui-ci avec le fleuve Lukashi et forma une musumba à Kakonde ku Buila. Il décida de rester caché là tant qu'il n'aurait pas trouvé un lieu où s'établir, par peur de connaître le même sort que Nkongolo Mwana face à Nsomwe.

Un jour, alors que ses hommes chassaient, l'un d'eux découvrit du vin de palme dans la forêt. Ils en burent tous et l'aîné du groupe leur dit d'attacher un morceau de viande de l'antilope qu'il avait tuée au tronc de l'arbre abattu. Ces palmiers appartenaient à Kaka Kitoto (titre de Kulu, la fille de Nsomwe). Kema (titre) est celui qui a la tâche d'en extraire le vin et c'est lui qui découvrit ce qui s'était passé et qui en portait la responsabilité. Ayant rapporté cela à Kaka Kitoto, celleci invita 'es Baluba et offrit quatre moutons au chef Mpibwe. Les hommes mangèrent et burent du vin de palme. Kaka Kitoto ordonna alors au ntshikala (l'attaché à la distribution) de montrer aux hommes, à l'exception de Mpibwe qu'elle décida de garder avec elle, les demeures où ils pourraient dormir. Elle chargea aussi son lamine (gardien des biens du chef) de placer le kipo (bracelet de perles bleues; ce terme signifie également collier) à la main gauche de Mpibwe, lui annonçant ainsi leur mariage. Celui-ci accepta et envoya un message à Ngoy Mulo qui fut heureux des 
événements car un moyen d'acquérir une terre s'était présenté tout seul.

Kaka Kitoto et Mpibwe Kitenge eurent deux enfants : Mueni Kitenge et Ngoie Kitenge. Par la suite Kaka Kitoto invita son grand-père, Nsomwe Olengiele, pour qu'il salue son mari. Nsomwe se sentit insulté d'avoir été appelé par un homme plus jeune que lui et refusa. Kaka Kitoto persuada alors Mpibwe de l'éliminer. La guerre fut déclarée entre les parties après que Lukunga et Kipasula (des messagers) furent revenus annoncer le refus de Nsomwe d'abandonner le muipata.

Mpibwe fut vainqueur, il tua Nsomwe, le décapita et enterra sa tête à Mpuluila. Cette guerre, appelée «le ronflement provenant du bosquet», dispersa les Kalebwe. Suite à leur victoire, Kaka Kitoto donna à Mpibwe Kitenge un tabouret de chef (lupuna sulu) et le fit Yakitenge Kuibwe.

Kalunguisha ka Mulopwe de Musengie annonça à Ilunga Ilunga a Mbidi Kiluwe que Mpibwe avait épousé sa grand-mère (classificatoire), Ilunga Ilunga jura alors que, jusqu'à sa mort, jamais il ne se risquerait à le voir. C'est la raison pour laquelle Yakitenge Kuibwe et Mulopwe de Musengie ne peuvent jamais se rencontrer.

Mpibwe et Kaka Kitoto ne restèrent pas longtemps ensemble, car cette dernière se mit à écouter les moqueries du peuple à propos de leur union. Un jour elle interrogea son mari sur ses origines. Mpibwe lui répondit qu'il ne connaissait pas très bien sa famille parce qu'il avait grandi en compagnie de ses oncles et que, depuis qu'il était devenu chasseur, il n'était jamais retourné chez ses parents. Kaka en fut déçue et déclara que ce seraient les enfants de Ngoy Mulo qui lui succéderaient et non pas ceux de son union avec Mpibwe. Elle quitta alors Mpibwe et alla s'installer à 
Ekungu en tant que chef. Mpibwe kitengie conserva le muipata jusqu'à sa mort et, celle-ci survenue, les Kalebwe appelèrent Kaka Kitoto qui fit de Ngoie Mulo le nouveau Yakitenge. (Hersak 1986, 12-5).

Dix ans pius tôt, un ingénieur belge, $\mathrm{K}$. Plasmans, avait recueilli une version fort semblable de ce récit auprès du même Kitumbika. Cette version a l'intérêt de mettre en évidence la dichotomie politique de la société songye en général et kalebwe en particulier. Elle s'ouvre en effet sur les deux généalogies qui vont fonder la société kalebwe : celle de Muilombe et Kaswa Makulo qui est à l'origine des différents sous-groupes kalebwe ainsi que de populations voisines, et celle de Kiobobo au sujet de laquelle nous apprenons que, si l'un de ses fils engendra la catégorie politique des bamfumu chez les Songye, les deux autres n'eurent pas une descendance moins prestigieuse : le chasseur Ndala engendra les Luba centraux et le nganga Kalombo les Luba occidentaux.

Le récit kalebwe attire ainsi notre attention sur les liens qui peuvent unir les sociétés luba et songye. Or il se fait que les Songye et les Luba, et particulièrement les Luba centraux, sont fort proches sur le plan linguistique (Bastin et al. 1983, $189 \&$ 195) et culturel; de plus l'histoire conquérante du royaume luba a souvent entraîné les chefferies songye dans son tourbillon (Reefe $1981,111-2,115-7 \&$ 149-50).

On retrouve aussi ce cousinage dans la structure même des gestes de fondation politique des Kalebwe et des Luba centraux comme va nous le montrer une lecture en miroir de celles-ci. Pour ce faire il me sera bien sûr difficile d'ignorer le travail de Luc de Heusch qui, dans Le roi ivre, analyse longuement la geste luba, aussi est-ce à partir de certaines de ses analyses que je me propose de lire le récit kalebwe.

Les trois héros de la geste luba ont pour nom Nkongolo, Mbidi Kiluwe et Kalala Ilunga, on retrouve l'écho de ces noms 


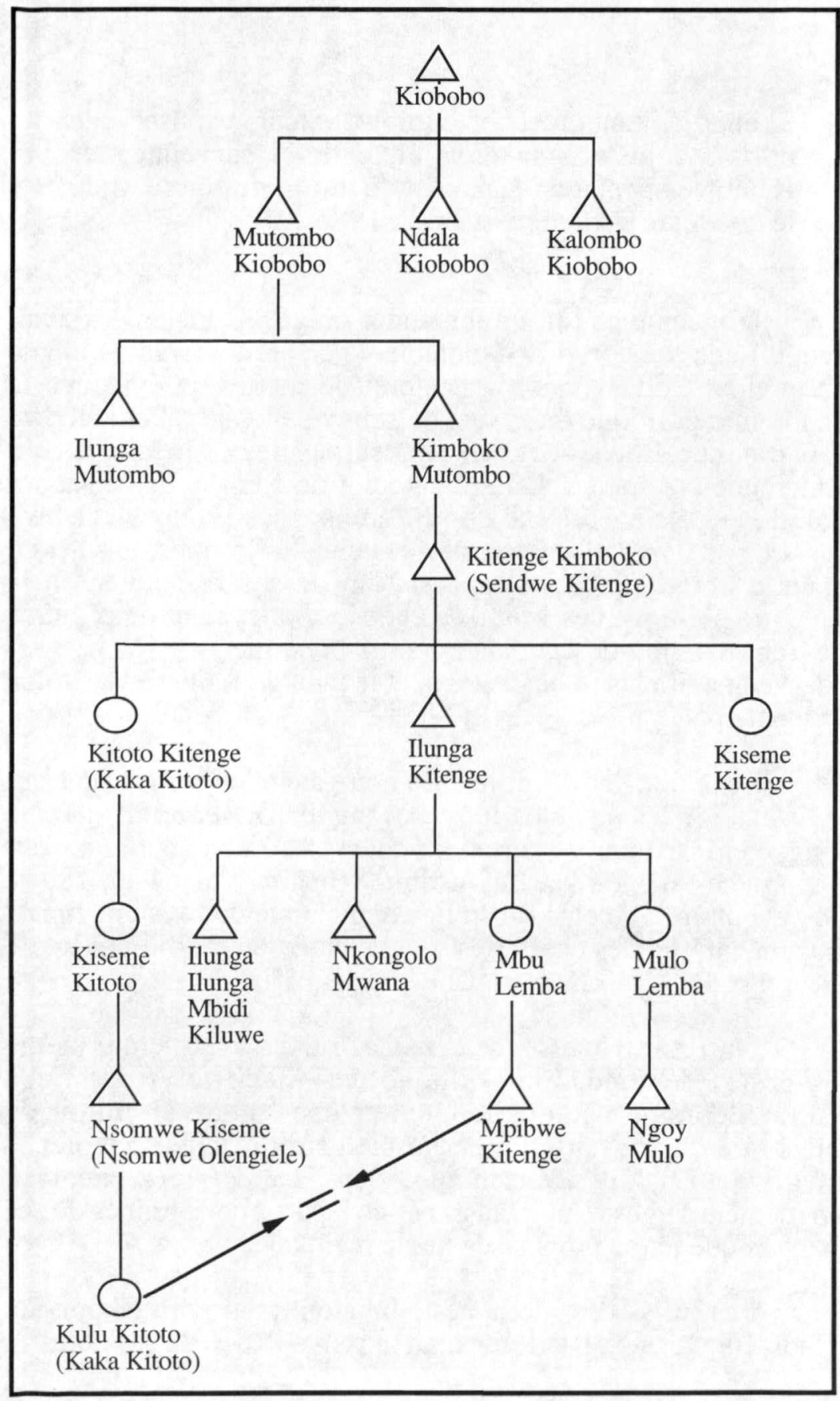

D'après hersak 1986, 13. 
dans ceux des deux fils d'Ilunga Kitenge : Ilunga Ilunga Mbidi Kiluwe et Nkongolo Mwana. Intéressons-nous par conséquent, le récit luba d'un côté, le récit kalebwe de l'autre, à ces deux frères dont les noms semblent la clé du lien qui unit les deux récits.

Ainsi que nous l'apprend la version Burton, les aventures que connaît Mbidi Kiluwe dans la geste luba sont dues à une imprudence : "le chasseur Ilunga Kiluwe régnait sur le Bupemba (sans doute le Buhemba de l'est). Il avait deux fils, Mbidi Kiluwe et Ndala, et une fille Mwanana qu'il chérissait. Devenu vieux, Ilunga Kiluwe souhaita que celle-ci lui succédât, bien que ses sujets eussent choisi Mbidi Kiluwe. Mwanana possédait un lion domestiqué. L'animal s'échappa tandis que Mbidi Kiluwe jouait avec lui. Furieuse Mwanana menaça de faire tuer son frère s'il ne rattrapait pas le lion. Mbidi Kiluwe fut contraint de poursuivre l'animal (...). Il perdit la trace du lion au fleuve Lualaba" (Heusch 1972, 24). Ainsi commencèrent les aventures de Mbidi Kiluwe qui partit en direction de l'ouest où il épousera les soeurs incestueuses de Nkongolo.

Il faut rapprocher cet épisode du récit luba de celui de la geste kalebwe où Sendwe Kitenge sacrifie sa propre fille et bannit son fils llunga Kitenge parce que celui-ci a manifesté sa désapprobation. Ilunga Kitenge part alors vers l'est, traverse le Lualaba et conquiert les Hemba. Si l'on ajoute à cela qu'Ilunga Kitenge est un forgeron tandis que Mbidi Kiluwe est un chasseur, une homologie inverse des deux récits se dessine :

\begin{tabular}{|lll|}
\hline SONGYE & L & LUBA \\
pays natal à l'ouest & U & pays natal à l'est \\
(Buhemba) & & \\
forgeron & A & chasseur \\
fille sacrifiée & L & fille chérie \\
conquête (guerre) de l'est & A & mariage à l'ouest \\
(Buhemba) & B & \\
& A & \\
\hline
\end{tabular}


Le fleuve Lualaba, que les deux fils exilés traversent en sens inverse, constitue la charnière de ce renversement. Nous retrouverons bientôt ce fleuve qui, rappelons-le, marque la limite orientale du pays songye.

Notons que le sacrifice impromptu de sa fille par Sendwe Kitenge prend tout son sens dans cette lecture en miroir : il répond à l'amour excessif que portait le père de Mbidi Kiluwe à sa fille.

Dans le récit luba, Mbidi Kiluwe, après avoir traversé le Lualaba, arrive chez Nkongolo, épouse les deux soeurs incestueuses de ce dernier tout en montrant des manières de roi, puis, suite à une dispute, repasse le Lualaba en sens inverse pour revenir dans son pays natal où son père est mort et son frère comme sa soeur absents.

On peut continuer de suivre parallèlement les aventures de Mbidi Kiluwe et des héros du récits kalebwe. Dans ce dernier toutefois, le retour est le fait des fils d'Ilunga Kitenge. En effet, les habitants du Buhemba renvoient Ilunga Ilunga Mbidi Kiluwe, celui-là même qui porte le nom du héros luba, de l'autre côté de la Lualaba à la recherche de son père. Cette recherche n'aboutit pas, Ilunga Ilunga s'arrêtant en chemin. Il revient alors à Nkongolo Mwana de continuer ce retour vers le pays natal.

C'est en suivant Nkongolo Mwana dans ses pérégrinations que l'on va retrouver de nombreux mythèmes de la geste luba. Ainsi c'est également sous le signe de l'eau que les femmes d'Ilunga Ilunga d'une part, de Nkongolo d'autre part, président à la rencontre des deux frères d'une part, de Mbidi Kiluwe et Nkongolo d'autre part. Le point d'eau comme lieu de rencontre entre des femmes autochtones et un étranger, généralement un chasseur, est un mythème extrêmement répandu dans la région; on le retrouve notamment dans le mythe d'origine politique eki (Fairley 1978, 110). Il semble se retrouver de façon identique dans les deux gestes qui nous occupent, si ce n'est que, dans le cas kalebwe, il préside à la réunion de deux frères, alors 
que dans le cas luba, Mbidi Kiluwe et Nkongolo sont des personnages que tout oppose.

Par la suite nous retrouvons une lecture en miroir des deux récits. Dans la geste luba, Nkongolo, à qui les exploits et la popularité grandissante de Kalala Ilunga, le fils de Mbidi Kiluwe, portaient ombrage, décide de le supprimer : "il tenta de l'attirer dans un piège à l'occasion d'un concours de danse. Au cours de la fête, Kahia, le tambourineur personnel de Kalala, découvrit le léger effondrement de terrain qui marquait l'emplacement d'une fosse dissimulée par une natte. Tandis que son maître évoluait dangereusement sur la piste aménagée, il l'avertit du danger en langage tambouriné. Kalala découvrit la ruse de Nkongolo en perçant le sol de sa lance. Il bondit alors comme une bête fauve au-dessus de l'assistance" (Heusch 1972, 26) pour rejoindre son père par delà le Lualaba que Nkongolo ne pourra jamais traverser. On fait évidemment immédiatement le rapprochement entre cet épisode et celui qui voit la rencontre de Nsomwe et Nkongolo Mwana dans le mythe kalebwe. On peut schématiser l'homologie inversée de ces deux versions d'un même mythème de la façon suivante si l'on se place du côté des deux Nkongolo:

\begin{tabular}{|cc|}
\hline SONGYE & LUBA \\
Nkongolo Mwana & Nkongolo \\
& \\
- prétendant au pouvoir & /- détenteur du pouvoir \\
- victime de l'épisode du trou /-organisateur de l'épisode \\
masqué par une natte & du trou masqué par une natte \\
- la ruse réussit & /- la ruse échoue \\
\hline
\end{tabular}

Dans les deux récits, cet épisode est suivi par un affrontement entre les deux protagonistes et, chaque fois, c'est le manieur de lance, symbole de son habileté guerrière, qui l'emporte, Kalala dans un cas, Nsomwe dans l'autre ${ }^{10}$. Il s'ensuit

10 Il convient de remarquer que la pluie est une alliée de Nsomwe (elle efface les traces du piège) comme elle est l'alliée de Kalala (L. de Heusch note que les 
qu'en dépit de ce qui distingue les deux gestes, le destin tragique de Nkongolo Mwana n'a rien à envier à son homologue luba : ayant échoué dans sa tentative de reprendre l'epata fondée par son grand-père, il retourne chez son frère qui le chasse et meurt en route, c'est-à-dire sur une terre qui n'appartient à personne (Hersak 1986, 16-7), près du fleuve Lualaba qu'il comptait traverser pour regagner son pays natal, le Buhemba. On retrouve donc ce fleuve, à chaque fois infranchissable pour ces figures de l'échec que sont les Nkongolo luba et songye.

Guerrier victorieux face à Nkongolo Mwana, Nsomwe connaît pourtant à son tour une fin tragique : il est défait par ce chasseur devenu guerrier triomphant qu'est Mpibwe Kitenge. Décapité par ce dernier, sa tête est enterrée à Mpuluila. Cette fin n'est pas sans comparaison avec celle du Nkongolo luba qui, tout au long de ses aventures, a successivement pour adversaire le chasseur Mbidi Kiluwe et le guerrier triomphant Kalala Ilunga, ce dernier en venant à bout et lui coupant la tête, celle-ci est engloutie par une termitière qui communique sa couleur rouge au paysage environnant. Luc de Heusch a établit une affinité entre la tête de Nkongolo et le soleil (Heusch 1972, 50-2) qui permet d'associer la tête sanglante et décapitée de Nkongolo au soleil déclinant et rougeoyant. Le mythe kalebwe, si l'on veut bien accepter l'homologie entre la fin du Nkongolo luba et celle du Nsomwe kalebwe, vient confirmer cette affinité. En effet, Mpibwe enterre la tête de son rival à Mpuluila. Il s'agit d'une rivière située à proximité de l'epata que le candidat Yakitenge ne peut traverser qu'à l'occasion de son investiture, encore faut-il qu'il la traverse à reculons et à gué. Cela fait, la mort viendra sanctionner tout nouveau passage; aussi, son mandat achevé, le Yakitenge contourne-t-il celle-ci pour quitter l'epata. De telles précautions ne sont pas vaines quand on sait que tout Yakitenge qui meurt à l'epata est enterré dans cette rivière dont on détourne momentanément le cours pour l'occasion. Si un Yakitenge meurt dans son village après avoir achevé son mandat, on enterre

eaux tumultueuses du Lualaba engloutissent l'armée de Nkongolo comme "un fleuve en crue au plus fort de la saison des pluics" (Heusch 1972, 53)). 
néanmoins dans la Mpuluila un sac rempli de feuilles de palmier qui le représentent (Pierrot 1938, 16, 18 \& 21). Les Songye comparent leurs chefs au soleil : nguba ekuilu, "soleil au zénith", disent-ils pour désigner le chef qui exerce son mandat, et de comparer celui-ci au soleil déclinant lorsque, son mandat achevé, il doit quitter l'epata pour céder sa place au nouveau titulaire. Le nom de la rivière Mpuluila semble renvoyer à cettte métaphore : en kiluba, -pùlwila est la forme applicative d'un verbe, -pùluka, qui signifie "tomber d'une certaine hauteur; descendre" et ce terme est utilisé pour décrire le mouvement descendant du soleil : "dyuba diba puluka : le soleil descend (depuis midi jusqu'au soir)" (Van Avermaet 1954, 545)"

Cette lecture conjointe des récits luba et songye montre clairement combien une même structure jalonne les deux récits. Il ne faut toutefois pas réduire le récit kalebwe à cette lecture en miroir de la geste luba. Il possède d'autres thèmes en commun avec les récits d'origine de cette région du Zaïre, telle, par exemple, toute la séquence qui décrit la rencontre de Mpibwe Kitenge et Kulu Kitoto, elle correspond point par point à la rencontre du chasseur luba Tshibinda Ilunga avec la princesse autochtone Lueji dans le mythe de fondation de l'Etat lunda (Duysters 1958, 82-3).

L'analyse structurale d'un récit révèle ainsi sa nature d'objet bricolé. Jeu sur et assemblage original de mythèmes communs à toute une aire culturelle, le mythe relève de la forme avant de relever du sens. D'où son efficacité : il n'est pas lié à une idéologie mais se pare de celle qu'on veut bien lui prêter. Le mythe n'est pas une explication qui rend compte mais une métaphore qui évoque. A ce titre, il abandonne la précision de la première aux discours exégétiques pour garder la souplesse de la seconde.

11 La pauvreté des dictionnaires songye existants (Samain s.d.; Stappers s.d.) nous a obligé à recourir à l'excellent dictionnaire kiluba de Van Avermaet; un informateur kalebwe m'a cependant confirmé que ce verbe existait dans leur langue avec le même sens. 
Un exemple va me permettre d'illustrer mon propos. On a vu que l'affrontement de Nsomwe Oliengele et de Nkongolo Mwana autour d'une fosse masquée d'une natte se retrouvait dans le récit luba. En fait on retrouve une telle scène dans de nombreux mythes de la région. Les Lumbu par exemple, un groupe luba oriental, racontent en ces termes la mort de leur premier chef, Kiguha:

Comme celui-ci aimait trop son fils et faisait tous ses caprices au détriment de la population, les neveux utérins de Kiguha projetèrent de le supprimer. Pour ce faire ils creusèrent, là où Kiguha avait l'habitude de venir se reposer, une fosse qu'ils garnirent de lances et recouvrirent d'une natte. Lorsqu'ils vinrent prendre quelque repos, Kiguha et son fils tombèrent dans le piège et y périrent. Voilà pourquoi le pouvoir se transmet désormais d'oncle maternel à neveu utérin et non de père en fils chez les Lumbu.

(Sha Banza Buludu,Nkomba et al., Luata, le 22 août 1991).

On peut schématiser ainsi la structure de ce mythème : une chute dans un orifice ouvert vers le haut (d'un objet, d'une personne) est congrue à l'opposition entre deux prétendants à deux pouvoirs politiques de nature différente, généralement associés respectivement à la filiation patrilinéaire et matrilinéaire. Evidente dans la mythologie des Luba orientaux, on retrouve cette opposition dans le récit kalebwe (Dunja Hersak a bien montré qu'un affrontement entre les lignées patrilinéaire et matrilinéaire de Sendwe Kitenge sous-tend cette partie du récit kalebwe (Hersak 1986, 17)) et il n'en va pas différemment dans le récit des Luba centraux (Heusch 1972, 102).

Ce mythème est un contenant puisqu'il peut accepter différents contenus : il peut tout aussi bien prôner la patrilinéarité que la matrilinéarité, faire triompher tel ou tel type de pouvoir. On voit donc que sa nature est formelle; à ses utilisateurs d'y placer 
un contenu. Luc de Heusch voulait-il dire autre chose quand, dans sa conclusion au Roi îvre, il écrivait à propos des mythes qu'ils s'échangent comme des marchandises mais qu'ils n'ont à proprement parler aucune valeur, ou encore qu'échappant à la fonction idéologique que les rois s'efforcent toujours de leur faire jouer, ils n'ont pas de maîtres (Heusch 1972, 299) ?

Dans les sociétés d'Afrique centrale rien n'est plus légitime que la tradition, or celle-ci relève de la répétition, c'est pourquoi l'usage répété des mythèmes dans les mythes fonde la légitimité de ceux-ci. Dès lors, formels (contenant) et légitimes, les mythes légitiment à leur tour les discours idéologiques qui les ancrent dans la société qui les véhicule. C'est pourquoi ils sont la manière légitime de conter une histoire politique.

Outre sa parenté structurale avec la geste luba, un autre aspect remarquable du récit kalebwe est l'opposition que l'on retrouve tout au long de celui-ci entre la figure du forgeron et celle du chasseur.

On sait toute la place qu'accordent les mythologies luba et lunda à ces chasseurs fondateurs de royauté que sont Mbidi Kiluwe et Tshibinda Ilunga ${ }^{12}$; ce sont également à des chasseurs, ou quelques fois à des guerriers, que les chefferies songye attribuent l'origine de leurs structures politiques ${ }^{13}$. En ce qui concerne le forgeron, Pierre de Maret (1985) a montré combien était fréquente son association au pouvoir en Afrique centrale. La geste luba elle-même, qui fait pourtant la part si belle au chasseur, connait aussi son forgeron : après avoir vaincu Kongolo, Kalala Ilunga dépose les armes pour apporter l'art de la forge dans tout

12 Chasseur se dit kiluwe en luba et tshibinda en lunda (Womersley 1984, 8).

13 Il est à noter que les mythes d'origine des entités politiques de cette région ne content pas la constitution d'une entité politique mais d'un ordre politique nouveau, ainsi Kalala Ilunga, par exemple, ne fonde pas un royaume (celui-ci en tant qu'entité politique lui préexiste) mais la royauté (il instaure de nouveaux usages politiques). 
le pays luba, il développe par la même occasion l'agriculture (Womersley 1984, 19-22) ${ }^{14}$. Les trois figures du chasseur, du guerrier et du forgeron se succèdent donc dans cet ordre dans la geste luba. On les retrouve selon un même ordre dans la geste de la chefferie songye des Eki où au chasseur qui fonde le nouvel ordre politique, succède un guerrier qui accroît la chefferie puis un forgeron qui y introduit son art et de nouvelles cultures (Fairley 1978, 110-41). Dans la geste kalebwe par contre, où l'on retrouve également ces trois figures associées au pouvoir, chasseur et forgeron s'affrontent à plusieurs moments du récit et, à chaque fois, le premier prendra l'avantage sur le second.

Dès le début du récit, la destinée forgeronne de la lignée cheffale kalebwe s'affirme; ainsi, quand les trois fils de Kiobobo ont à se partager ces trois spécialisations, c'est le métier de forgeron que choisit l'ancêtre de Kitenge Kimboko. A la génération suivante, l'opposition entre les fonctions de chasseur et de forgeron est encore plus claire : des deux fils qu'engendrera Mutombo Kiobobo, l'un, Ilunga sera 'chasseur d'eau' (pêcheur) et l'autre, Kiomboko, forgeron; à nouveau c'est le forgeron qui perpétuera la lignée cheffale kalebwe. La suite du récit kalebwe voit en effet les deux fils de Mutombo Kiobobo se disputer un coquillage blanc kilase; on pourrait penser que celui-ci devrait revenir à Ilunga qui a fait profession de les 'chasser' mais son père dit à Kimboko le forgeron, qui l'a trouvé, de le conserver comme une chose propre aux chefs. Sur ce les deux frères se brouillent et se séparent, Ilunga restera sans descendance tandis que Kiomboko engendrera Sendwe Kitenge. Une séquence identique ouvre la généalogie Kayaye publiée par Moeller (1936, 144); Wynants, qui l'a récoltée, nous dit que "presque toutes les populations du territoire de Kasongo placent à l'origine de leur migration une guerre résultant d'une contestation au sujet de la propriété d'un «kiziwa»" (Ibidem, 145).

14 Si la version Burton de la geste luba fait du père de Mbidi Kiluwe un chasseur (Burton 1972, 4), la version Womersley en fait, elle, un forgeron (Womersley 1984, 1). 
Il faut rapprocher cet épisode du récit kalebwe du rituel d'intronisation d'un chef chez les Luba centraux où, pour être investi du pouvoir, le candidat doit obtenir le consentement des esprits et vaincre à la guerre. Le signe que les ancêtres acceptent le candidat à la succession du chef défunt est généralement un objet blanc ramené d'un milieu aquatique : "le [futur] roi (ou son champion) entreprend un voyage dans la résidence aquatique des anciens esprits du royaume pour conclure avec eux une alliance. La preuve tangible de cette alliance est un objet blanc rapporté par le pélerin" (Petit 1992-1993, 432). Chez les Kilembwe, une chefferie songye au nord de Lubao, le droit d'être investi chef politique est symbolisé par la détention d'une boule de kaolin (Mwanana Kasongo a Kisanga, Kitenge, le 21 octobre 1993). Des pratiques similaires se rencontrent dans les rituels d'investiture des chefs politiques chez les Kalebwe. Lors de l'investiture d'un chef de province, des marques de terre blanche ntoshi sont faites sur le corps du nouveau chef politique (Pierrot 1937, 11-3) et, lors de l'intronisation d'un nouveau Yakitenge, une mère de jumeaux "prend de la terre blanche (toshi) et en jette sur la poitrine et le dos du candidat [Yakitenge] en faisant pour lui des voeux" (Ibidem, 16). Le ntoshi désigne dans la langue songye le kaolin, il s'agit d'une argile blanche qui est extraite du lit d'une rivière (Hersak 1986, 185), autrement dit un objet blanc issu d'un milieu aquatique... A la lumière de ces informations, on voit toute l'importance symbolique de l'épisode du coquillage dans le récit kalebwe.

C'est également un couple chasseur-forgeron qui clôture le récit, Kulu Kitoto, l'épouse du chasseur Mpibwe Kitenge, est en effet associée au monde de la forge. Le "forgeron Musonge utilise pour forger et façonner des pièces, deux marteaux de tailles inégales. Le plus grand se nomme nyindu, le plus petit kitoto, ce dernier servant à parachever la pièce. Le nyindu est l'homme, kitoto, la femme" (Wauters, 1949 : 119). Par conséquent, face à Mpibwe Kitenge, Kulu Kitoto rappelle par 
son nom ${ }^{15}$ son appartenance à la lignée forgeronne de Sendwe Kitenge. Incontestablement la cheffesse domine dans ce couple : c'est elle qui décide de leur mariage, c'est elle qui renie leur descendance. Aussi l'attitude autoritaire de Kulu Kitoto dans une société gérée par les hommes, comme le fait que Kiomboko ait obtenu un coquillage issu des 'terres de chasses' de son frère, dit clairement la prédominance du forgeron sur le chasseur.

On ne peut comprendre ce que recouvre exactement cette opposition entre la figure du forgeron et celle du chasseur qu'en s'intéressant à la pensée et à la structure politique kalebwe dont cet antagonisme est en fait une métaphore.

Si la plupart des chefferies songye comptent quelques milliers d'habitants à peine, ce n'est cependant pas le cas de la chefferie des Kalebwe qui en compte de deux à trois dizaines de milliers. Cette différence notable s'accompagne d'une plus forte hiérarchisation politique : alors que l'accès à la fonction politique suprême est direct dans les chefferies de petite taille, dans celle des Kalebwe, qui est divisée en provinces et sous-provinces, pour accéder au titre politique suprême (le buyakitenge), le candidat doit au préalable avoir été investi, toujours selon le principe songye de la rotation du pouvoir, comme chef de sa sous-province puis de sa province. Ce cheminement politique plus long est évidemment à mettre en rapport avec la taille, tant géographique que démographique, de la chefferie ${ }^{16}$.

Les Kalebwe symbolisent les titres de chef de province et sous-province par la figure du chasseur. On dit d'un tel chef qu'il est le descendant de Mpibwe Kitenge, que c'est un chasseur et que le forgeron est le père des chasseurs, en ce sens qu'il fournit

15 Avec ceux des quatre enfants d'Ilunga Kitenge (au rang desquels figurent les deux frères Ilunga Ilunga Mbidi Kiluwe et Nkongolo Mwana), les noms de Kulu Kitoto et Mpibwe Kitenge sont les seuls dans la descendance de Kiobobo à déroger à la règle qui préside à leur formation : nom personnel + nom du père.

16 L'exégèse politique songye laisse à penser qu'une telle organisation résulterait de l'association, forcée ou volontaire, de plusieurs chefferies de taille plus modeste. 
des armes de fer aux chasseurs (Pierrot 1938, $10 \& 12$ ). Par contre, le chef suprême est, lui, associé au forgeron. Alors que c'est d'un arc dont on fait usage dans le rituel d'investiture des chefs de province, c'est une enclume qui est utilisée dans celui d'un nouveau Yakitenge, et le lukunga, héraut des premiers occupants du pays, dit au nouveau Yakitenge : "si tu voyages tu ne pourras loger que dans une forge (eyasu) ou sous le toît qui abrite le haut fourneau (elungu)" (Ibidem, 17).

Les Kalebwe disent que le chasseur est un léopard et le forgeron un lion, le premier étant le cadet du second ${ }^{17}$. On retrouve une conception similaire dans un récit tiré du bukishi, l'initiation eki qui s'est diffusée en territoire kalebwe. Le chasseur Kitenge, un léopard, est l'enfant adultérin de Kankumbe, un pygmée (un twa), et Kitoto Mbu, femme d'Efile (Dieu); c'est le chef de la forêt (Wauters 1949, 176), un chef qui approvisionne et dont l'insigne est la viande. Mais Dieu ne reconnaît pas cet enfant et en fait lui-même un à sa femme : le forgeron Kitenge, le lion, "qui cultive le maïs, s'il est épuisé - le millet. Kitenge, l'Ainé de toutes les plaines" (Ibidem, 319); son insigne du pouvoir est un anneau rouge ${ }^{18}$ qui lui donne un droit d'aînesse sur le léopard.

La pensée songye conceptualise donc l'antagonisme du chasseur et du forgeron dans les termes suivants : chasseur, léopard, cadet, forêt / forgeron, lion, aîné, plaine. Mais le récit du bukishi pense aussi l'opposition du chasseur et du forgeron en termes alimentaires. Si le chasseur dispense la viande, le forgeron, lui, est associé à l'agriculture. Nous avons vu

17 Il est pourtant un autre animal qui est leur aîné à tous deux, c'est la civette, nshima. La civette cst associée au cite de la capitale, le représentant des premiers occupants du sol, qui en porte la peau. Lorsque des dignitaires voyagent ensemble, c'est celui qui a le statut le plus élevé qui ferme la marche. Aussi, le léopard ouvret-il la marche, suivi du lion et enfin de la civette (Pierrot 1938, 22). Car ne l'oublions pas c'est en faveur des premiers occupants du sol que le chasseur et le forgeron apportent leurs bienfaits, en échange de quoi ceux-ci les investissent du pouvoir politique.

18 Probablement un anneau de métal (fer ou cuivre). 
qu'effectivement, que ce soit chez les Kalebwe, les Eki ou les Luba centraux, le forgeron apporte toujours de nouvelles plantes à cultiver en même temps que l'art de la forge. On a donc également l'opposition 'forgeron, agriculture / chasseur, viande de chasse' et le fait que les Songye associent chasseurs et pêcheurs (que l'on pense à Ilunga, le chasseur d'eau) parce qu'ils ne cultivent pas (Moeller 1936,151) en apporte la confirmation.

Lors de l'investiture d'un Yakitenge, il lui est dit que désormais il n'est plus un vagabond, un chasseur, un léopard (Pierrot 1938, 25). La métaphore est la suivante : le candidat au buyakitengie a traversé toute la structure politique kalebwe, étant successivement chef d'une sous-province, d'une province et, enfin, de la chefferie toute entière. Désormais qu'il n'est plus un chasseur mais un forgeron, sa quête du pouvoir est achevée ${ }^{19}$. Les Kalebwe opposent ainsi la mobilité du chasseur-léopard en quête de pouvoir à la tranquillité du forgeron-lion qui occupe le pouvoir suprême.

Le chasseur marqué du signe de l'errance est un thème récurrent de la mythologie de cette région d'Afrique centrale. Il faut rattacher cette errance au fait que ces chasseurs fondateurs d'un nouvel ordre politique sont dits Luba, le verbe kuluba signifie en effet errer, être perdu (Colle 1913, 1; Makonga 1948, 308; Petit 1993, 41). Dans le mythe kalebwe cependant, les forgerons n'errent pas moins que les chasseurs et si, en installant son epata, Kitenge met fin à son errance, le récit associe clairement ce dernier à un Luba en vertu de celle-ci. Cette réunion du chasseur et du forgeron sous le signe de l'errance se rencontre également dans une autre région, celle de Bukama (Luba centraux), où les chefferies sont fondées soit par un chasseur à la poursuite d'un gibier soit par un forgeron en quête de minerai

19 On peut comparer l'organigramme de la chefferie et les lieux d'activité du forgeron et du chasseur. Le forgeron travaille dans le village tandis que le chasseur opère en brousse, comme le Yakitenge est au centre du système politique -et sa capitale au centre de la chefferie- tandis que les chefs subalternes sont en périphérie. 
(Verellen 1952). A la lumière de cette information, il semble bien qu'il faille dissocier les différentes étapes du travail de la forge et, en particulier, la quête du minerai de son travail. La recherche du minerai n'est pas sans comparaison avec la chasse, à l'image de cette dernière elle repose sur la chance quand le gisement est petit ou mal connu. C'est pourquoi les spécialistes qui prospectent le minerai sont souvent qualifiés de chasseurs; c'est notamment le cas pour les Mikebwe, une peuplade située au nord-est du pays songye (Kita cité par Maret, sous presse). Par conséquent il convient de faire la distinction entre l'errance qui caractérise le forgeron à la recherche de minerai et la sédentarité du forgeron qui fond ou qui forge comme Kitengie à l'epata. De cette manière, on peut réduire la dissonance mise à jour entre le rite et le mythe. Quoi qu'il en soit, le rite et le mythe n'en partagent pas moins un message commun : l'errance prend fin une fois arrivé à l'epata, car, dans le récit kalebwe, dès l'instant où Sendwe Kitenge fonde l'epata l'errance n'est plus le fait que des chasseurs. Ainsi, s'ils ne se répètent pas, le mythe et le rite se répondent tout au moins ${ }^{20}$.

On trouvera dans le tableau suivant la synthèse des qualités antagonistes du chasseur et du forgeron :

\begin{tabular}{|lll|}
\hline $\begin{array}{lll}\text { Forgeron } \\
\text { Produits agricoles }\end{array}$ &, $\begin{array}{c}\text { Chasseur } \\
\text { Produits de la chasse (et de } \\
\text { Lion }\end{array}$ & la pêche) \\
Plaine & $/$ & Léopard \\
Sédentarité & $/$ & Forêt \\
\hline
\end{tabular}

Ainsi c'est par l'usage original d'éléments récurrents de l'imaginaire politique de cette partie de l'Afrique que les Kalebwe conceptualisent leur structure politique particulière. Mais s'il est

20 Pour une comparaison entre le mythe et le rituel d'investiture du Yakitenge : Hersak 1986, 17-20. Sur les rapports entre rites et mythes dans la région : Petit 1993, 531-3. 
original dans un contexte régional, cet usage des personnages du chasseur et du forgeron n'en reste pas moins cohérent dans le cadre de la société songye qui ne les restreint pas à cet antagonisme politique. Dans le cas du forgeron, un aspect particulièrement remarquable de sa richesse symbolique apporte sur ce personnage singulier un éclairage qui nous fait mieux comprendre pourquoi c'est lui que les Kalebwe ont placé au sommet de leur structure politique.

Il existe chez les Songye comme dans bon nombre de sociétés d'Afrique centrale une "homologie sur le plan symbolique entre le rôle créateur de la femme et du métallurgiste" (Maret 1980, 277). Le forgeron songye utilise deux marteaux de tailles différentes symbolisant respectivement l'homme et la femme (Wauters 1949, 119; confer supra), aussi le produit de son travail s'apparente-t-il au fruit de l'union d'un homme et d'une femme. Dans le cadre du bukishi, les Songye disent du chemin qui mène les récipiendiaires au lieu d'initiation où ils vont symboliquement renaître, que c'est le "chemin emprunté par les hommes vers la forge, c'est-à-dire la naissance" (Wauters 1949, 141). A plusieurs reprises d'ailleurs, dans les textes du bukishi recueillis par Wauters, le terme 'forger' est utilisé pour signifier la corception d'enfants (Ibidem, 216 \& 357). Les parties génitales féminines, quant à elles, sont "le feu de la forge" (Pierrot 1938, 9). Dans un mythe bala, une chefferie songye voisine des Kalebwe et qui fut autrefois leur tributaire (Ibidem, annexe $n^{\circ}$ IV), Mulopwe Kamusenge, premier homme et assistant de Dieu, était assis sur une grosse pierre avec sa femme. A minuit, il se mit à créer instantanément toutes les populations du monde; il disait "cinquante personnes là-bas, cinquante personnes ici" et elles apparaissaient ${ }^{21}$ (Merriam 1974, 31). On retrouve une séquence identique dans un mythe kalebwe (Weydert 1938, 119-21). A n'en pas douter Mulopwe Kamusenge, l'assistant de

21 Son oeuvre créatrice achevée, Mulopwe se mit à jouer du tambour kyondo, dans un autre mythe, c'est caché dans un tel tambour (porté par un chasseur) que Sendwe kitenge arrive chez les Songye (Moeller 1936, 450-1). 
Dieu $^{22}$, et sa femme forgent la race humaine sur cette grosse pierre; le récit bala nous dit en effet qu'il y avait du sang sur celleci, le sang que perd une femme juste avant de mettre un enfant au monde (Merriam 1974, 106) tandis que la version Plasmans de la geste kalebwe nous indique qu'il faut voir dans cette pierre la métaphore d'une masse-enclume. Cette version parle en effet de "Yakitengie assis sur une pierre, (...) la pierre où l'on forge les flèches" (Plasmans s.d., III. 19). On se souvient en effet que le premier Yakitenge établit sa forge et son epata à ebwe, qui signifie rocher en songye. Wauters est encore plus explicite : "Sendwe Kitenge choisit pour l'emplacement d'une forge un rocher qui émergeait du milieu de la rivière. Le rocher lui servait d'enclume" (Wauters 1916, 10) ${ }^{23}$. L'association entre ce rocher, le sang lié à la conception et la forge se retrouve encore dans un petit récit ésotérique lié à la geste kalebwe rapporté par Pierrot $(1938,22-3)$.

La lune est un symbole de fertilité dans la pensée songye (Hersak 1986, 26; Merriam 1974, 105; Wauters 1949, 234) ${ }^{24}$ et le cycle lunaire est tout à la fois associé aux périodes conceptionnelles de la femme et aux cycles agricoles (Hersak 1986, 134; Wauters 1949, 231). Il n'est donc pas étonnant qu'un lien unisse celle-ci à un forgeron. On trouve en effet dans la cosmologie des Kalebwe et des Eki un curieux personnage, Sende Mudimi ${ }^{25}$, le forgeron-agriculteur qui cultive au moyen de l'éclair (Wauters 1949, 235-6), or celui-ci n'est autre que l'enfant que la lune eut de son épouse Nkwaba (Ibidem, 235), l'étoile du soir (Merriam 1974, 106).

22 Un dicton songye dit : "Vous cherchez le divin sans succès, cependant vous voyez le forgeron dans sa forge" (Wauters 1949, 125).

23 On retrouve ces informations de Wauters dans Moeller $(1936,451)$.

24 Il est dit dans le récit bala que Mulopwe Kamusenge se mit à créer à minuit, c'est-à-dire en pleine nuit, domaine de la lune par opposition au jour, domaine du soleil.

25 Certains Kalebwe font de Nsendwe Mudimi le propriétaire de l'epata de Yakitenge-kui-Ebwe (Plasmans 1966, 1). 


\section{Problèmes africains contemporains}

Comme nous l'indique ce bref survol, le contexte symbolique de la figure du forgeron déborde largement le cadre politique et ce point de vue plus global nous éclaire d'autant mieux sur sa place dans ce cadre. Métaphore de la fertilité humaine, fondateur de la fertilité agricole, le forgeron est lié aux aspects les plus fondamentaux de la vie songye. Faut-il dès lors s'étonner que, chez les Kalebwe, il soit aussi à l'origine de la structure politique ? Peut-être faut-il d'ailleurs inverser la proposition, le politique n'aime-t-il pas à se présenter comme à l'origine de tout et de la vie sociale en particulier ? C'est ce que semble signifier l'assimilation fréquente en Afrique centrale du héros civilisateur et du fondateur politique.

J'ai dit précédemment que le mythe est la manière légitime de conter l'histoire, j'ajouterai qu'à ce titre il légitime le groupe qui le véhicule en tant que groupe. Nous avons vu que les Kalebwe sont constitués de plusieurs entités qui participent d'un même système politique et que, par leur mode d'accès au titre suprême, ces entités se fondent en un tout. En effet, ce n'est qu'après avoir été investi chef de sa sous-province puis de sa province que le candidat pourra accéder au buyakitenge; les Kalebwe disent que le chasseur cesse alors de vagabonder pour devenir forgeron. On voit donc que ce qui fonde l'unité kalebwe, c'est cette capitale commune vers laquelle tous convergent, mais c'est aussi et surtout le récit qui l'instaure : l'histoire de Sendwe Kitenge et de Mpibwe Kitenge, le forgeron et le chasseur, qui instituèrent l'ordre politique qui est le leur aujourd'hui. 


\title{
BIBLIOGRAPHIE
}

\author{
BASTIN, Y., A. COUPEZ, B. de HALLEUX
}

Classification lexicostatistique des langues bantoues (214 relevés). Bulletin des Scéances de l'Académie Royale d'Outre-Mer, 27 (2) : 173-199. 1983.

BOONE, O.

Carte ethnique du Congo. Quart Sud-Est. Tervuren : Annales du Musée Royal de l'Afrique Centrale. 1961.

BURTON, W. F.P.

Luba religion and magic in custom and belief. Tervueren : Annales du Musée Royal de l'Afrique Centrale. 1961.

COLLE, P.

Les Baluba. Bruxelles: A. Dewit. 1913.

DUYSTERS, L.

Histoire des Aluunda. Problèmes d'Afrique Centrale, 12 (40) : 79-98. 1958.

FAIRLEY, N.J.

Mianda ya Ben'Ekie : a history of the Ben'Ekie. Ph.D. Diss. State University of New York, Stony Brook. 1978.

HERSAK, D.

Songye Masks and Figure Sculpture. Londres : Ethnographica. 1986.

HEUSCH, L. de

Le roi ivre ou l'origine de l'Etat. Paris : Gallimard. 1972.

Rois nés d'un coeur de vache. Paris: Gallimard. 1982.

MAKONGA, B.

Samba-a-Kya-Buta. Bulletin des Juridictions Indigènes et du Droit Coutumier Congolais, $10: 304-316.1948$.

MARET, P. de

Ceux qui jouent avec le feu : la place du forgeron en Afrique centrale. Africa , 50 (3) : 263-279. 1980 .

The smith's myth and the origin of the Ieadership in Central Africa. In African Iron Working - Ancient and Traditional, eds. R. Haaland and P. Shinnie, 73-87. Bergen : Norwegian University Press. 1985.

Metallurgies, metamorphoses and metaphors. In Iron, master of them all, eds. A. Roberts \& W. Dewey. Sous presse.

MERRIAM, A. P.

An African World. The Basongye Village of Lupupa Ngye . Bloomington \& Londres : Indiana University Press. 1974.

MOELLER, A.

Les grandes lignes des migrations des Bantous de la Province Orientale du Congo Belge. Bruxelles : Institut Royal Colonial Belge. 1936.

PETIT, P.

Rites familiaux, rites royaux. Etude du système cérémoniel des Luba du Shaba (Zaïre). Thèse de Doctorat en Sciences Sociales, Université Libre de Bruxelles. 1993. 


\section{PIERROT, P.}

Note préliminaire. Archives de la Zone de Lubao (Zaïre). 1938.

PLASMANS, $\mathrm{K}$.

Histoire des Bekalebwe. Archives de Kongolo (Zaïr). S.d.

Histoire de la famille Yamudimi. Archives de Kongolo (Zaïre), 1966.

REEFE, T. Q.

The Rainbow and the Kings. A History of the Luba empire to 1891. Berkeley, Los Angeles \& Londres : University of California Press. 1981.

SAMAIN, A.

La langue Kisongye : grammaire - vocabulaire - proverbes. Bruxelles : Goemare. S.d.

STAPPERS, L.

Vocabulaire Songye. Archives du Musée Royal de l'Afrique centrale, Section Linguistique. S.d.

VERELLEN, C.

Notes sur la région de Kapamai du territoire de Bukama. Archives de la SousRégion du Haut Lomami. Fond Reefe. 1952.

VAN A VERMAET, E. (en collaboration avec B. MBUYA)

Dictionnaire kiluba - français. Tervueren : Annales du Musée Royal du Congo Belge. 1954.

WAUTERS, C.

Notes sur les traditions basonge. Archives du Musée Royal de l'Afrique Centrale, Section Histoire. 1916.

L'ésotérie des Noirs dévoilées. Bruxelles : Editions Européennes. 1949.

WEYDERT, J.

Les Baluba chez eux. (Polycopié). 1938.

WOMERSLEY, $\mathrm{H}$.

Legends and Histotry of the Luba . Los Angeles : Crossroads Press. 1984. 